\title{
Substanzielle und prozedurale Gerechtigkeit in der Verteilung von Gesundheitsgütern'
}

\author{
Claudia Landwehr
}

\section{Substantial and procedural justice in the distribution of health goods}

Abstract: When health care resources become scarce, their distribution turns into a problem of distributive justice. The paper starts by addressing the question which particular properties of health care goods are relevant for their allocation before assessing whether distributional conflicts in health care can be solved by recourse to abstract principles of justice. It becomes clear that the contextualisation of abstract principles involves severe problems, while at the same time consensus on eligible principles or priority rules between them is not to be expected. What is at issue thus is the development of fair decision-making procedures in the distribution of health goods - but here, too, normative criteria of procedural justice are insufficient as long as institutional design choices are not empirically informed on the distributive consequences of specific properties of procedures and institutions.

Keywords: Theories of Justice, Health Care Goods, Distributional Conflicts, DecisionMaking Procedures

Schlagwörter: Gerechtigkeitstheorie, Gesundheitsgüter, Verteilungskonflikte, Entscheidungsverfahren

\section{Einleitung}

„Gesundheit ist nicht alles, aber ohne Gesundheit ist alles nichts. “2 Auch wenn chronisch Kranke oder behinderte Menschen die Allgemeingültigkeit dieser Aussage bestreiten werden, so ist Gesundheit ohne Frage ein Gut mit besonderen Qualitäten. Güter, die wie Gesundheit eine Voraussetzung dafür darstellen, wesentliche Lebensziele überhaupt mit Aussicht auf Erfolg verfolgen zu können, werden als konditionale, transzendentale oder schlicht als Grundgüter bezeichnet (Gosepath 2007, S. 20). Ihr besonderer, grundlegender Charakter ist ein Argument dafür, Strukturen, die ihre Gleichverteilung - soweit möglich - befördern, als öffentliches Gut zu realisieren. Aber auch wenn der Anspruch auf öffentliche Sicherstellung eines Systems der Gesundheitsversorgung, das die Gesundheit der Bürger schützt, erhält und im Krankheitsfall nach Möglichkeit wiederherstellt, in

1 Ich danke Karl Marker, Annette Schmitt, Jürgen Sirsch, Doris Unger, Katharina Böhm und zwei anonymen Gutachtern für die PVS für hilfreiche Kommentare und Anregungen zu diesem Artikel.

2 Dieser zu Unrecht Arthur Schopenhauer zugeschriebene Aphorismus ist in Alltagsgesprächen ein Allgemeinplatz. Der Schopenhauer-Forscher Matthias Koßler weist auf die falsche Zuschreibung des Zitats hin. Belegt ist die Verwendung durch den Hamburger Arzt und Jazz-Musiker Abbi Hübner. 
Deutschland wie in praktisch allen anderen Industrienationen realisiert ist, bleiben unter Knappheitsbedingungen Verteilungskonflikte und Entscheidungszwänge bestehen. ${ }^{3}$

Dieser Aufsatz geht zunächst der Frage nach, ob praktische Probleme in der Allokation knapper Gesundheitsgüter durch die Anwendung substanzieller normativer Verteilungsprinzipien gelöst werden können und müssen, oder ob hier allein Kriterien prozeduraler Gerechtigkeit einen Maßstab darstellen können. Wenn wir zur Bewertung von Allokationsentscheidungen auf prozedurale Gerechtigkeitskriterien zurückgreifen müssen, so stellt sich im Anschluss die zentrale Frage, worin solche bestehen können und ob sich auf dieser Grundlage ein gerechtes Entscheidungsverfahren konkret definieren ließe. Ich werde im Folgenden den besonderen Charakter der Güter Gesundheit und Gesundheitsversorgung kurz darlegen und dabei argumentieren, dass unter Knappheitsbedingungen Entscheidungen über die Rationierung von Gesundheitsgütern unausweichlich sind. Im Anschluss werde ich den Versuch, empirische Verteilungskonflikte um knappe Gesundheitsgüter durch die Anwendung abstrakter substanzieller Verteilungsprinzipien zu lösen, diskutieren und aufzeigen, warum dieser Versuch an der Komplexität der Praxis scheitern muss - und faktisch bereits gescheitert ist. Schließlich werde ich im zentralen Teil dieses Beitrags Kriterien prozeduraler Gerechtigkeit in der Verteilung von Gesundheitsgütern und ihre Anwendung diskutieren. Ich komme dabei zu dem Schluss, dass die normative Benennung von Kriterien eines gerechten Verfahrens, wie sie für den Bereich der Gesundheitsversorgung durch Norman Daniels und James E. Sabin (Daniels u. Sabin 2002; Daniels 2008) überaus einflussreich geschehen ist, für die demokratische Verfahrensauswahl unzureichend ist: diese muss durch Untersuchungen über die Verteilungswirkungen konkreter Verfahrensmerkmale empirisch informiert sein.

\section{Gesundheit und Gesundheitsversorgung als besondere Güter}

Was folgt aus dem besonderen, weil konditionalen und transzendentalen Charakter des Gutes Gesundheit? Für John Rawls (Rawls [1971] 1999, S. 62) ist Gesundheit ein „natürliches Primärgut“. Für Martha Nussbaum zählen „not dying prematurely“ und „being able to have good health” zu den zentralen menschlichen capabilities. Eine Gesellschaft, die diese nicht in hinreichendem Maße garantiere, könne keine gerechte sein (Nussbaum 2006, S. 74-76). Zugleich ist klar, dass eine Gleichverteilung des Gutes Gesundheit im Sinne der Angleichung des Gesundheitszustandes aller Mitglieder einer Gemeinschaft nicht möglich ist. Als „natürliches Gut" ist sie zunächst einmal ungleich verteilt. Menschen kommen mit unterschiedlichen Gesundheitschancen auf die Welt, und schwere Krankheiten oder Verletzungen, die sie als Schicksalsschläge ereilen, können oftmals auch mit

3 Ein System, das Gesundheit selbst oder ihre Gleichverteilung garantiert, ist nicht denkbar. Es kann also nur um ein System der Gesundheitsversorgung gehen, wobei vorausgesetzt wird, dass diese der Gesundheit der Anspruchsberechtigten dient. Die USA stellen mit ihrem schwach ausgebauten öffentlichen Gesundheitssystem im Vergleich der Industrienationen eine Ausnahme dar, doch auch hier ist die Notfallversorgung sowie eine Absicherung der Ärmsten flächendeckend gewährleistet. 
fortgeschrittenster medizinischer Technologie nicht oder nicht vollständig geheilt werden. Auch wenn diese Ungleichheit selbst noch keine Ungerechtigkeit darstellt, liegt es nach Rawls an der Gesellschaft, mit der Ungleichheit gerecht umzugehen: „The natural distribution is neither just nor unjust. [...] What is just or unjust is the way that institutions deal with these facts" (Rawls [1971] 1999, S. 102). ${ }^{4}$

Gerechtigkeitsfragen stellen sich also nicht bei der Verteilung des Gutes Gesundheit, sondern bei der Verteilung von Gütern, die der Erhaltung und Wiederherstellung dieses Gutes dienen, also Gütern der Gesundheitsversorgung. Rawls zählt eine angemessene Gesundheitsversorgung zwar nicht zu den sozialen Primärgütern, deren gerechte Verteilung von vorrangiger Bedeutung ist. Wie Norman Daniels argumentiert, ist eine solche jedoch zur Sicherstellung des sozialen Primärguts fairer Chancengleichheit (fair equality of opportunity) unerlässlich (Daniels 2008, S. 57). Doch bei Rawls lässt sich noch ein weiteres Argument finden, das eine Absicherung gesundheitlicher Lebensrisiken begründen könnte: Rawls gesteht ein, dass dieselben Freiheiten unter unterschiedlichen Lebensumständen unterschiedlichen Wert besitzen können (Rawls [1971] 1999, S. 179). Auch wenn hierin keine Einschränkung der Freiheiten selbst bestehe, müsse das Differenzprinzip den geringeren Wert, den die Grundfreiheiten für einige Mitglieder der Gemeinschaft besitzen, kompensieren. Die Sicherheit, im Bedarfsfall unabhängig von Status und Vermögen Anspruch auf medizinische Hilfe zu haben, kann eine solche Kompensation darstellen, die den Wert der Freiheit für Benachteiligte erhöht.

Fehlende Gesundheit kann Menschen also nicht nur an der Verfolgung wesentlicher Lebensziele hindern, sondern auch daran, demokratische Partizipationsund bürgerliche Freiheitsrechte effektiv zu nutzen. Sofern ein Teil der Bevölkerung aufgrund ihres Gesundheitszustandes an der Nutzung dieser Rechte gehindert ist, steht auch deren für den demokratischen Rechtsstaat zentrale Gleichverteilung in Frage. Bei T. H. Marshall (1893-1981), dessen Theorie sich als Begründung des britischen Beveridge-Modells einer universellen Grundsicherung lesen lässt, ist die angemessene Absicherung gesundheitlicher Lebensrisiken in diesem Sinne weniger als Menschenrecht denn als „soziales Bürgerrecht“ zu verstehen (siehe Marshall [1949] 1992). Soziale Rechte auf Bildung, Gesundheitsversorgung und ökonomische Grundsicherung stellen nach Marshall eine Voraussetzung für die effektive Nutzung von Freiheits- und politischen Rechten und damit für die wirksame Inklusion aller Bürger dar. Durchaus ähnlich wie Marshall sieht auch der libertäre Theoretiker Kliemt die instrumentelle Bedeutung öffentlicher Gesundheitsvorsorge für die Funktion des demokratischen Rechtsstaates: „Öffentlich garantierte Teilhaberechte an Gesundheitsleistungen finden im pluralen Rechtsstaat ihre Rechtfertigung [...] nicht im Zweck der „Gesundheit“, sondern in der Wahrung des Kernbestandes der liberalen Rechtsordnung selber." (Kliemt 2003, S. 69).

Die Forderung, dass ein demokratischer Rechtsstaat, zumal ein wirtschaftlich hochentwickelter, ein Mindestmaß an Gesundheitsversorgung sicherstellen soll,

4 Die Tatsache, dass auch die Menschenrechtserklärung der Vereinten Nationen in Art. 25 ein Recht auf Gesundheitsversorgung benennt, ist zumindest ein Hinweis auf den hohen Stellenwert, den diese im Gerechtigkeitsempfinden der Menschen weltweit einnimmt. 
scheint über alle politischen Lager und akademischen Schulen hinweg geteilt zu sein und kaum einer Verteidigung zu bedürfen. Dennoch ist es wichtig, sich die Argumente vor Augen zu führen, mit denen dieselbe Forderung jeweils begründet wird. Auf der einen Seite - bei Rawls, Daniels und Nussbaum sowie in der Menschenrechts-Konzeption - wird die Versorgung mit elementaren Gesundheitsgütern als nicht zurückweisbare Gerechtigkeitsforderung angesehen, die auf dem politischen Prozess und Diskurs enthobenen Gerechtigkeitsprinzipien beruht: für Rawls, Daniels und Nussbaum sind dies, trotz der Unterschiede zwischen ihren Theorien, letztlich Prinzipien der fairen Chancengleichheit, in der Menschenrechtskonzeption naturrechtlich-transzendental begründete Rechte auf Unversehrtheit.

Auf der anderen Seite - beim sozialdemokratischen Theoretiker Marshall ebenso wie beim libertären Denker Kliemt - geht es darum, die Funktionsbedingungen des demokratischen Rechtsstaats aufrecht zu erhalten. Marshall beschreibt im optimistischen Ton der Nachkriegsjahre die historisch-kausale Entwicklung des modernen Nationalstaats als Komplettierung und Vervollkommnung der Bürgerrechte: Die Ausweitung negativer Freiheitsrechte ermöglicht die Ausweitung positiver Partizipationsrechte, über die wiederum soziale Bürgerrechte, wie das Recht auf Gesundheitsversorgung, demokratisch errungen werden. Die Gewährleistung sozialer Bürgerrechte ermöglicht dann die effektive Nutzung und damit Sicherung von demokratischen und Bürgerrechten - ein ,Engelskreis‘. Bei Kliemt geht es schlicht um die Sorge vor der Empörung der Bevölkerung, die durch eine NichtVersorgung kranker Menschen ausgelöst werden und systemerodierendes oder -umstürzendes Potenzial haben könnte.

Aber auch angesichts der Einigkeit darüber, dass ein System der Gesundheitsversorgung als öffentliches Gut zu realisieren ist, bleibt als zentrale Frage offen, welche Gesundheitsgüter in diesem System jedem zur Verfügung gestellt (oder, in ökonomischer Terminologie, unterhalb marktläufiger Preise angeboten) werden sollen, und wie diese Gesundheitsgüter zu verteilen sind. Neben der besonderen Bedeutung, die aus ihrer Funktion zum Erhalt und zur Wiederherstellung des konditionalen Gutes der Gesundheit selbst erwächst, haben Gesundheitsgüter weitere besondere Eigenschaften, die für ihre Verteilung relevant sind. Viele der im Gesundheitswesen konkret zu verteilenden Güter sind zunächst einmal nur begrenzt teilbar: Auch wenn die verwendeten Ressourcen teilbar sind - das dringend benötigte Spenderorgan oder auch das einzelne Krankenhausbett sind es nicht. Gesundheitsgüter sind zudem heterogene Güter. Das heißt, dass es von großer Bedeutung ist, wer wann welche Leistung erhält, da der Nutzen einer Leistung mit dem Empfänger und seiner Erkrankung variiert (Elster 1995). Eine Blinddarmoperation etwa stellt nur dann einen Nutzen dar, wenn der Blinddarm tatsächlich entzündet ist. Wenn nicht, bedeutet sie im Gegenteil einen Schaden für den Patienten.

Die Tatsache, dass es sich bei Gesundheitsgütern um heterogene Güter handelt, ist grundlegend für das Informationsproblem, das Entscheidungen über ihre Verteilungen aufwerfen, denn: Wer sagt uns, unter welchen Bedingungen eine Allokation Nutzen generiert und unter welchen nicht? Zudem sind Informationen hochgradig asymmetrisch verteilt: Der Arzt, also Leistungserbringer, weiß mehr über den Bedarf des Patienten, also des Leistungsempfängers, als dieser selbst. Die 
Nachfrage des Patienten nach Gesundheitsleistungen ist damit zum großen Teil extern gesteuert, was Marktversagen wahrscheinlich macht.

Offene Fragen und Entscheidungsnotwendigkeiten finden sich in einem öffentlich kontrollierten System der Gesundheitsversorgung dabei auf mehreren Ebenen: Welchen Anteil seiner öffentlichen Ressourcen soll ein Staat für Gesundheitsversorgung ausgeben? Wie ist das Gut Gesundheit gegen Güter wie Bildung, Kultur oder Grundsicherung zu gewichten? ${ }^{5}$ Das für Gesundheitsversorgung auszugebende Budget kann zunächst von den benötigten beziehungsweise nachgefragten medizinischen Leistungen und ihrem Preis abhängig gemacht werden. Diese Strategie verfolgte man in der Zeit der Expansion des Wohlfahrtsstaates, also bis in die 1970er-Jahre, in den meisten Industriestaaten. In Deutschland erhielt diese Politik, mit Bezug auf die gesetzliche Krankenversicherung, die Bezeichnung „ausgabenorientierte Einnahmepolitik“. Mit dem Rückbau sozialer Sicherungssysteme angesichts stagnierenden Wirtschaftswachstums und sinkender Einnahmen aus Steuern und Sozialversicherungsbeiträgen, aber auch in Anbetracht steigender Gesundheitsausgaben wurde diese Politik durch ihr Gegenteil, eine „einnahmeorientierte Ausgabenpolitik“ ersetzt. Solange Entscheidungen darüber, welche Ressourcen eine Gesellschaft für die Gesundheitsversorgung zur Verfügung stellt, unmittelbar an den im Gesundheitssystem entstehenden Bedarf gekoppelt waren (wie in Zeiten der ausgabenorientierten Einnahmepolitik), konnten Verteilungskonflikte innerhalb des Gesundheitssystems vermieden werden. Wenn Entscheidungen über die Verteilung von Ressourcen auf einzelne gesellschaftliche Subsysteme aber von den Entwicklungen innerhalb dieser Systeme, und in diesem Fall des Gesundheitssystems, entkoppelt werden (müssen), werden hier „tragic choices" notwendig - Entscheidungen über die Verteilung von Gesundheitsgütern unter Knappheitsbedingungen, also Rationierungsentscheidungen (vgl. Calabresi u. Bobbit 1978).

Auch wenn man Argumente, die sich auf Probleme auf der Einnahmeseite des Gesundheitssystems beziehen - schwindende oder wegbrechende Steuer- und Beitragseinnahmen - in Frage stellen kann (siehe etwa Kühn 2004), hat die Entwicklung der Ausgaben für Gesundheitsleistungen zweifelsohne eine Dynamik, die explizite Grenzziehungen unvermeidlich macht. Zentral sind hier der technologische Fortschritt und die Innovationsfreude der pharmazeutisch-technischen Industrie, die tagtäglich neue Patente anmeldet, in erster Linie auf Arzneimittel. Viele neue Medikamente ermöglichen dabei nicht die Heilung von Krankheiten, sondern verbessern im Wesentlichen die Lebensqualität und -erwartung der Patienten, müssen also über viele Jahre eingenommen und finanziert werden.

Der technologische Fortschritt entwickelt jedoch erst in Verbindung mit einem weiteren, im Konsum von Gesundheitsgütern grundlegenden Problem eine entsprechende Dynamik auf der Ausgabenseite: Viele Gesundheitsgüter haben keinen negativen Grenznutzen, sondern immer einen zumindest potenziellen positiven Zusatznutzen. Für Operationen und Behandlungen mit schweren Nebenwirkungen mag dies nicht gelten, wohl aber für eine Vielzahl nebenwirkungsarmer Medi-

5 Dem noch vorgelagert ist natürlich die Frage nach dem angemessenen Anteil öffentlicher Ausgaben an den Gesamtausgaben einer Volkswirtschaft. 
kamente und für diagnostische Verfahren. So lassen sich an einem beschwerdefreien und augenscheinlich gesunden Patienten zahllose Untersuchungen durchführen, bei denen die Möglichkeit besteht, dass sie zur Entdeckung schwerwiegender und behandlungsbedürftiger Krankheiten führen. ${ }^{6}$ Die Informationsasymmetrie zwischen Anbieter und Konsumenten, also zwischen Arzt und Patient, ermöglicht zudem eine angebotsinduzierte Nachfragesteigerung: Der Patient kennt seinen eigenen Bedarf an Gesundheitsleistungen weniger gut als der Arzt, weshalb der Arzt über sein Informationsverhalten die Nachfrage des Patienten gezielt steuern kann. Der Konsum von Gesundheitsgütern ist deshalb - insbesondere dann, wenn diese wie in öffentlichen Systemen zunächst kostenlos zur Verfügung gestellt werden - tendenziell „exzessiv“, was bedeutet, dass Patienten mehr konsumieren als sie benötigen (Aaron u. Schwartz 1990). Die Kombination von anhaltender technologischer Innovation und exzessivem Konsum von Gesundheitsgütern führt daher zu einer Dynamik des Kostenanstiegs, der ohne Grenzziehungen nicht beizukommen ist.

Wenn wir Ressourcen unter Knappheitsbedingungen verteilen müssen, also nicht alles, was gewünscht und gebraucht wird, auch finanziert werden kann, nach welchen Prinzipien soll dann die Allokation dieser knappen Ressourcen erfolgen? Mit Norman Daniels gefragt: „How can we meet health needs fairly when we can't meet them all? “ (Daniels 2008, Kap. 4). Da, wie ich im Folgenden argumentieren werde, Gerechtigkeitsprinzipien in der Verteilung zwar eine zentrale Rolle spielen, diese aber miteinander konkurrieren und sich nicht selbst anwenden, sondern vielmehr eine Kontextualisierung und Gewichtung erfordern, ist die entscheidende Frage, wer wie über die Verteilung knapper Gesundheitsgüter entscheiden soll, letztlich praktisch-politischer Natur.

\section{Substanzielle Gerechtigkeitsprinzipien in praktischen Verteilungskonflikten}

Bei der Suche nach einer gerechten Verteilung knapper Gesundheitsgüter bieten umfassende normative Gerechtigkeitstheorien, wie etwa der Utilitarismus oder der Egalitarismus, mögliche Verteilungsprinzipien und sogar konkrete Lösungsvorschläge an. Die Rationierung von Gesundheitsleistungen ist in der praktischen Philosophie und Gerechtigkeitstheorie, insbesondere im angloamerikanischen Raum, als lebensnahes Beispiel zur Illustration praktischer Implikationen der jeweiligen Theorie beliebt. Die Fälle, mit denen hier gearbeitet wird, sind jedoch in der Regel hypothetischer Natur - etwa die Verteilung des Arzneimittels, mit dem man einen Erkrankten heilen oder zehn Gesunde impfen könnte. Fraglich bleibt, ob diese hypothetischen Verteilungskonflikte den faktischen hinreichend ähneln, um Lösungsvorschläge in die Praxis übertragen zu können: Lassen sich aus umfassenden Gerechtigkeitstheorien praktisch anwendbare Verteilungsprinzipien ableiten?

Ein Problem kann zunächst darin bestehen, dass sich viele Gerechtigkeitstheorien nicht, wie etwa der klassische Utilitarismus, auf ein einziges Gerechtigkeits-

6 Auch diagnostische Verfahren bergen Risiken, die aber von Ärzten und Patienten häufig nicht als solche anerkannt werden, etwa die psychische Belastung durch falsch positive Ergebnisse. 
und Verteilungsprinzip reduzieren lassen, sondern mehrere normative Prinzipien formulieren, für die Prioritätsregeln festgelegt werden müssen. Darüber hinaus sind normative Prinzipien für die Anleitung konkreter Entscheidungen in der Regel nicht hinreichend spezifiziert. Sie können vielleicht zur Bewertung von Verteilungsentscheidungen herangezogen werden, für die Auswabl einer der unzähligen denkbaren Verteilungslösungen sind über abstrakte Gerechtigkeitsprinzipien hinaus konkretere Verteilungsprinzipien erforderlich.

Auch hier bietet die gerechtigkeitsphilosophische Literatur Ansatzpunkte. Theoretisch begründete Prinzipien wie Gleichheit, Leistung, Anspruch oder Bedarf können jeweils für sich genommen die Verteilung knapper Güter leiten. Autoren wie Michael Walzer (Walzer 1984) und David Miller (Miller 1999) schreiben die unterschiedlichen Gerechtigkeitsprinzipien unterschiedlichen gesellschaftlichen „Sphären“ oder Interaktionsbereichen zu: Während das Leistungsprinzip auf dem Arbeitsmarkt legitim Geltung beansprucht, muss bei der Ausstattung mit bürgerlichen und demokratischen Rechten das Gleichheitsprinzip gelten und in Vertragsbeziehungen ein Anrechtsprinzip.

Für den Bereich der Gesundheitsversorgung liegt im Sinne einer solchen Sphären-Zuschreibung von Gerechtigkeitsprinzipien das Bedarfsprinzip nahe. Doch selbst bei einer solch eindeutigen Zuschreibung blieben Fragen hinsichtlich der Explikation und Operationalisierung des Bedarfsbegriffs offen: Wollen wir Bedarf im Sinne einer „rule of rescue“ (McKie u. Richardson 2003) schlicht als Dringlichkeit, gemessen an der wahrscheinlichen Überlebenszeit bis zum Tod, messen?? Oder wollen wir auch andere Formen von Bedarf anerkennen: den Bedarf nach Linderung oder Behebung von Schmerzen und Behinderungen, den Bedarf, gegen eine potenziell tödliche Krankheit geschützt (etwa geimpft) zu werden, den Bedarf, in Lebenskrisen psychologische Hilfe zu erhalten, oder auch den Bedarf einer Kinderwunschbehandlung? Wenn wir diese unterschiedlichen Bedarfe anerkennen, wie sollen wir sie im Konfliktfall gegeneinander abwägen: Finanzieren wir, unter Bedingungen knapper Ressourcen, eine Chemotherapie, die das Leben eines todkranken Krebspatienten um wenige Wochen verlängert, oder eine künstliche Befruchtung, die einen sehnlichen und belastenden Kinderwunsch erfüllt?

Spätestens hier stellt sich die Frage, inwieweit abstrakte normative Gerechtigkeitstheorien und die in ihnen vorgeschlagenen Verteilungsprinzipien konkrete politische Verteilungsentscheidungen instruieren können. Eine Gerechtigkeitsphilosophie, die für sich in Anspruch nimmt, konsensfähige Verteilungsprinzipien zu definieren, welche die politische Bewältigung und eigentliche Entscheidung in Verteilungskonflikten unnötig machen würden, erscheint gleichermaßen naiv und in anmaßender Weise realitätsfern. Und in der Tat würden die meisten Philosophen weder die Herausforderungen leugnen, die in der Kontextualisierung abstrakter Prinzipien bestehen noch bestreiten, dass der Erfolg von Lösungsvorschlägen in Verteilungskonflikten letztlich von ihrer gesellschaftlichen Akzeptanz abhängt.

Anders als die Philosophie müssen die Politikwissenschaft und auch die politische Theorie den gesellschaftlichen Wert- und Interessenpluralismus ebenso wie

7 Der MELD (Measure of End-Stage Liver Disease)-Score operationalisiert eine solche rule of rescue in der Vergabe knapper Spenderorgane zur Lebertransplantation. 
die Schwierigkeiten, die sich bei der Kontextualisierung abstrakter Prinzipien ergeben, als Grundbedingungen politischen Handelns und Entscheidens stets im Blick behalten. Aus dieser Sicht kann und darf es nicht nur um die Begründung und Verteidigung abstrakter Verteilungsprinzipien gehen. Vielmehr müssen im Sinne einer empirischen Gerechtigkeitsforschung und -theorie auch die Gerechtigkeitsvorstellungen der Bürger erkundet und politische Institutionen daraufhin untersucht werden, inwieweit sie mit diesen in Einklang stehen. ${ }^{8}$

Sowohl die Umfrageforschung als auch die Analyse politischer Diskurse zeigen, dass kein einzelnes Gerechtigkeits- oder Verteilungsprinzip als universell gültiges anerkannt wird oder auch nur das Denken und Diskutieren über Gerechtigkeit dominiert. Vielmehr findet sich eine breite Zustimmung zu unterschiedlichen Prinzipien der Verteilung wie Gleichheit, Leistung, Anrecht oder Bedarf (siehe Becker u. Hauser 2004; Liebig u. May 2009, S. 5). Diese sind für sich genommen jeweils begründungsfähig und werden vielfach sämtlich als zustimmungswürdig anerkannt. Probleme ergeben sich erst dann, wenn sie im konkreten Anwendungsfall miteinander in Konflikt geraten. So zeigt sich in der Praxis etwa, dass auch Menschen, die das Bedarfsprinzip als zentrales Verteilungsprinzip in der Gesundheitsversorgung anerkennen, in konkreten Fällen auf konkurrierende Prinzipien zurückgreifen. So verlangen im Sinne des Eigenverantwortungs-Diskurses viele Bürger, dass Raucher oder Übergewichtige für ihr (vermeintlich) unverantwortliches Gesundheitsverhalten zur Verantwortung gezogen werden, was der Anwendung eines Leistungsprinzips gleichkommt. Andere nehmen, gerade in Sozialversicherungssystemen wie dem deutschen, für sich ein aus Einzahlungen resultierendes und vom Bedarf unabhängiges Anrecht auf Gesundheitsleistungen - etwa eine Erholungskur - in Anspruch: hier kommt das Anrechtsprinzip zur Anwendung. Zudem betrachten die meisten Menschen sozial und regional ungleich verteilte Gesundheitschancen als Problem: Im Sinne von Gleichheitsprinzipien (Equity oder faire Chancengleichheit) muss es, unabhängig vom Bedarf, darum gehen, Ungleichheit zu reduzieren.

Vor allem aber konkurrieren Bedarfsprinzipien in konkreten Allokationsentscheidungen mit Effizienzprinzipien. Effizienz stellt für sich genommen kein eigenständiges Gerechtigkeitsprinzip dar, da sie kein eigenständiges Ziel bildet, das es um seiner selbst willen zu verwirklichen gilt. Auch wenn utilitaristische Verteilungslösungen in hypothetischen Beispielen oft auf spontane Ablehnung stoßen, so scheint ein abgemilderter Utilitarismus, der die Maximierung des gesellschaftlichen Gesamtnutzens zumindest als eines unter mehreren Kriterien zur Bewertung von Verteilungslösungen berücksichtigt, in der Bevölkerung doch weit verbreitet. Bürger befinden sich in Fragen der Gesundheitsversorgung immer in einer Doppelrolle: als Beitrags- bzw. Steuerzahler wünschen sie eine effiziente Verwendung ihrer Einzahlungen, als Patienten eine bedarfsorientierte Versorgung. Und auch wenn Effizienz nicht als um seiner selbst willen zu verfolgendes Ziel gesehen wird, so stellt ihr Gegenteil, die Verschwendung von Ressourcen unter Knapp-

8 Zur empirischen Gerechtigkeitsforschung und -theorie, siehe Liebig 2008. 
heitsbedingungen, für die Betroffenen doch in jedem Fall ein erhebliches Gerechtigkeitsproblem dar, das es zu beheben oder zumindest zu reduzieren gilt.

In der politischen Praxis konkurrieren also eine Vielzahl unterschiedlicher Interessen und Gerechtigkeitsprinzipien um Akzeptanz, wenn es um die faire Verteilung und Rationierung von Gesundheitsleistungen geht. Verteilungsentscheidungen erfordern die Gewichtung und Aggregation dieser Interessen und Wertvorstellungen. Es ist dabei aber keineswegs so, dass sich Interessen und Werte jeweils konkreten, abgrenzbaren gesellschaftlichen Gruppen zuschreiben lassen. Vielmehr ist die Interessenlage der Bürger - als Beitrags- und Steuerzahler, Patienten und Angehörige von Patienten - in den meisten Fällen ebenso komplex wie ihre Werthaltungen es sind. Die wenigsten Menschen sind gewillt, einem einzigen Gerechtigkeitsprinzip in allen Fragen Vorrang vor anderen zu geben. Vielmehr sehen sie in unterschiedlichen Bereichen und Situationen unterschiedliche Prinzipien als legitime Verteilungsgrundsätze an; und selbst im auf den ersten Blick eindeutigen Fall der Verteilung von Gesundheitsgütern stehen die benannten Prinzipien (und möglicherweise weitere) miteinander im Konflikt.

Die Schwierigkeiten, die sich in der Definition, vor allem aber in der Gewichtung und Aggregation von Verteilungsprinzipien ergeben, haben sich in der Verteilung von Gesundheitsgütern in der politischen Praxis eindrücklich gezeigt: Zahlreiche Länder haben in den 80er- und 90er-Jahren mit Expertenkommissionen den Versuch unternommen, Prinzipien für die Allokation knapper Ressourcen in der Gesundheitsversorgung zu definieren. Norwegen setzte 1985 die erste „Lønning Commission “ zur Prioritätensetzung in der Gesundheitsversorgung ein, Neuseeland initiierte 1992 ein umfassendes Bürgerbeteiligungsverfahren, und in den Niederlanden prägte der 1992 veröffentlichte Bericht der „Dunning Commission" den Diskurs. Einzig der US-Staat Oregon versuchte sich jedoch an der Definition einer expliziten Rangordnung von sogenannten condition-treatment pairs (Paaren von Gesundheitszuständen und entsprechenden Behandlungsmethoden), in den übrigen Fällen hoffte man über die transparente und konsensuelle Bestimmung von Grundprinzipien der gerechten Verteilung Richtlinien für Entscheidungen über konkrete Leistungen zu schaffen.

Diese Kommissionen, die ich als ,Prinzipienkommissionen' charakterisieren will, waren von einer zentralen Hoffnung getragen: der Hoffnung, dass über Grundprinzipien gerechter Verteilung ein gesellschaftlicher Konsens zu erreichen ist, der „tragische Entscheidungen“ (Calabresi u. Bobbit 1978) in Einzelfällen zwar nicht unnötig macht, sie aber doch, aufgrund ihrer Legitimation durch konsensuelle Prinzipien, ihrer Kontingenz und Schmerzhaftigkeit beraubt. Brisante politische Entscheidungen wären, wenn man sich einmal über grundlegende Prinzipien geeinigt hätte, aus denen Entscheidungen abzuleiten sind, nicht mehr notwendig: Informationen über Bedarf und Behandlungsmethoden könnten dann schlicht in einen ,Verteilungsautomaten' (in der Praxis: eine Expertenkommission, vielleicht sogar ein Computerprogramm) eingespeist werden, der durch Anwendung der konsensuell definierten Prinzipien und Kriterien eine Prioritätenliste errechnet.

Um die Jahrtausendwende setzte sich jedoch die Einsicht durch, dass ein Konsens über Gerechtigkeitsprinzipien allenfalls auf einer abstrakten Ebene möglich 
ist, auf der Prinzipien nicht miteinander in Konflikt geraten. Søren Holm verkündete für die nordischen Länder, die mit ihren Prinzipienkommissionen Vorreiter in der Rationierungsdebatte gewesen waren, ein "goodbye to simple solutions“ (Holm 2000). Mit dieser Feststellung, dass jede Einzelentscheidung über die Finanzierung einer Therapie zur Behandlung einer bestimmten Erkrankung, vielleicht sogar jede Entscheidung über die Finanzierung der Behandlung einer konkreten Erkrankung eines einzelnen Patienten, die erneute Prüfung und Gewichtung von Interessen und Gerechtigkeitsprinzipien erfordert, verschob sich der Fokus der Rationierungsdebatte von Gerechtigkeitsprinzipien und Verteilungskriterien auf die Verfahren und Gremien, mit denen Entscheidungen über die Finanzierung und Priorisierung medizinischer Leistungen in der politischen Praxis getroffen werden (ebd.). Gerechtigkeitsüberlegungen richten sich dann nicht mehr auf Verteilungsergebnisse, sondern darauf, wie diese zustande kommen, es geht also um Kriterien prozeduraler statt substanzieller Gerechtigkeit.

\section{Formen prozeduraler Gerechtigkeit}

Auch wenn Rationierungsentscheidungen in der Gesundheitsversorgung substanzielle Gerechtigkeitsvorstellungen ihrer Adressaten berühren, so bedeutet das Scheitern abstrakter substanzieller Prinzipien an der Komplexität der Empirie doch, dass Entscheidungsträger gewissermaßen auf sich selbst zurück geworfen werden, wenn Entscheidungen zu genuin politischen, weil kontingenten, Entscheidungen werden. Die Politisierung von Entscheidungen lässt sich mit Greven als ein Prozess oder Zustand beschreiben, in dem die Politik zum einzig anerkannten Normsetzer wird (Greven 1999). Normen lassen sich dem politischen Prozess also nicht (mehr) vorgeben und lassen sich auch nicht (mehr) legitim aus höherrangigen Prinzipien ableiten, sie werden erst in diesem Prozess gesetzt. Entsprechend muss sich das Augenmerk, wie von Holm betont, auf die Gerechtigkeit und Legitimität des politischen Entscheidungsprozesses richten. In der Theorie wie in der politischen Praxis wird die Hoffnung auf einen substanziellen Prinzipienkonsens also durch die Hoffnung darauf ersetzt, dass sich zumindest auf der Metaebene der Verfahrensentscheidung ein Konsens darüber finden lässt, was ein gerechtes Verfahren ausmacht.

Was aber kann Verfahrensgerechtigkeit oder prozedurale Gerechtigkeit in der Verteilung von Gesundheitsgütern bedeuten, und wie verhält sie sich zu substanziellen Gerechtigkeitskriterien? John Rawls hat eine Unterscheidung zwischen drei Formen beziehungsweise Begriffen der Verfahrensgerechtigkeit getroffen, die in diesem Zusammenhang zentral ist (Rawls [1971] 1999, S. 73-78). Er unterscheidet zwischen vollkommener Verfahrensgerechtigkeit (perfect procedural justice), unvollkommener Verfahrensgerechtigkeit (imperfect procedural justice) und reiner Verfahrensgerechtigkeit (pure procedural justice).

Vollkommene Verfahrensgerechtigkeit liegt Rawls zufolge dann vor, wenn zum einen ein unabhängiger (akzeptierter) Maßstab für die Gerechtigkeit von Verteilungsergebnissen vorliegt und das Verfahren zum anderen garantiert, dass seine Ergebnisse, gemessen an diesem Maßstab, gerecht sind. Berühmtes Beispiel hierfür sei das Teilen eines Kuchens. Wenn wir als Maßstab für Gerechtigkeit die Gleich- 
verteilung, also die gleiche Größe der Kuchenstücke ansehen, dann kann sie durch folgendes Verfahren garantiert werden: Die Person, die den Kuchen mit dem Messer teilt, ist als letzte an der Reihe, ein Stück zu wählen. Sie wird den Kuchen, soweit möglich, in gleich große Stücke schneiden, denn so wird das für sie selbst verbleibende Stück so groß wie möglich sein.

In politischen Kontexten sind Beispiele für vollkommene Verfahrensgerechtigkeit rar. Die Prinzipienkommissionen, die die frühen Jahre der Rationierungsdiskussion prägten, waren aber letztlich von einem Gerechtigkeitsverständnis geprägt, bei dem substanzielle und prozedurale Gerechtigkeit im Sinne von Rawls' vollständiger Verfahrensgerechtigkeit zusammenfallen: Erkennt man als Maßstab die konsensuell bestimmten Prinzipien an, so garantiert die exakte Ableitung konkreter Entscheidungen aus diesen eine gerechte Verteilung. Das Verfahren ist hier ein Prozess der deduktiven Ableitung, es hat keinen politischen Charakter und letztlich auch keinen Entscheidungscharakter: Eine Entscheidung, die nicht getroffen wird, sondern sich selbst trifft, ist keine Entscheidung.

Unvollkommene Verfahrensgerechtigkeit liegt nach Rawls dann vor, wenn zwar ein unabhängiger Maßstab für die Beurteilung von Ergebnissen vorliegt, das Verfahren aber nicht garantiert, dass eine diesem Maßstab entsprechende Entscheidung erreicht wird. Klassisches Beispiel für unvollständige Verfahrensgerechtigkeit sei eine Gerichtsverhandlung, bei der es um die Feststellung der Schuld des Angeklagten in einem Strafverfahren geht. Die Entscheidung des Gerichts ist dann richtig und korrekt, wenn ein Schuldiger verurteilt und ein Unschuldiger freigesprochen wird - lehnen wir diesen Maßstab ab, dann müssen wir das Gerichtsverfahren und das Strafrecht insgesamt ablehnen. Zugleich aber ist klar, dass Gerichte nicht immer in der Lage sind, die Schuldigkeit von Angeklagten korrekt festzustellen: Gelegentlich werden Schuldige freigesprochen und Unschuldige verurteilt. Das Gerichtsverfahren ist also als Verfahren unvollkommen - aber es ist dennoch das Beste, das uns zur Verfügung steht, weshalb wir seine Ergebnisse zumindest vorläufig akzeptieren. Zu beachten ist dabei, dass die Art von Gerechtigkeit, um die es in der Gerichtsverhandlung geht, nicht distributive, sondern (im Sinne von Aristoteles) korrektive Gerechtigkeit ist. Beim Urteil über Schuld oder Unschuld selbst geht es gar nicht um Gerechtigkeit, sondern um die Feststellung eines Sachverhaltes - und mit der (als objektiv und geteilt vorausgesetzten) Realität gibt es eben einen eindeutigen Maßstab. Aber lassen sich auch politische Entscheidungsprozesse als Fall unvollständiger Verfahrensgerechtigkeit begreifen und mit einem Gerichtsverfahren vergleichen?

Epistemische Demokratietheorien (etwa Estlund 1993; List u. Goodin 2001; Goodin u. Estlund 2004) verstehen die Demokratie zumindest auch als Methode zur „Entdeckung“ von Wahrheiten und gerechten Lösungen. Ein solches epistemisches Demokratieverständnis, das Rawls' Vorstellung unvollständiger Verfahrensgerechtigkeit entspricht, steht auch im Mittelpunkt deliberativer Demokratietheorien, obwohl einzelne Theoretiker das epistemische Element sehr unterschiedlich auslegen. Deliberative Demokratietheorien verorten das epistemische Potenzial der Demokratie in argumentativen Willensbildungsprozessen, die der Stimmabgabe und eigentlichen Entscheidung voraus gehen. Zentral ist hier das Geben und Nehmen von Gründen, der interaktive Prozess der Begründung und Rechtferti- 
gung nicht nur von Entscheidungsoptionen, sondern auch ihrer Prämissen. In einem solchen Prozess werden Entscheidungsoptionen, über deren Ungerechtigkeit oder negative Konsequenzen Einigkeit hergestellt werden kann, ausgeschlossen, und gemeinschaftlich Optionen definiert, die einer gerechten Lösung nahekommen. Es geht also nicht - wie in Rawls' Beispiel der Strafverhandlung vor Gericht - um die Wahrscheinlichkeit einer gerechten Entscheidung, sondern um einen Prozess der Annäherung an eine gerechte Entscheidung durch den Diskurs. Dieser Prozess ist, auch weil sich der Kontext, in dem Entscheidungen getroffen werden, beständig ändert, niemals abzuschließen, und wenn er durch eine Entscheidung unterbrochen werden muss (weil politische Entscheidungen eben drängen), dann ist das Ergebnis immer fehlbar. Der Diskurs wird nach der Entscheidung fortgesetzt und muss sie revidieren können.

Die Unabgeschlossenheit (und prinzipielle Unabschließbarkeit) deliberativer Diskurse und die Fallibilität ihrer Ergebnisse bedeuten jedoch nicht, dass der unabhängige Gerechtigkeitsstandard aufgegeben wird: Moralische Richtigkeit bzw. Gerechtigkeit transzendiert in epistemischen Demokratietheorien Rechtfertigung (vgl. Lafont 2003, S. 176). Viele Demokratietheoretiker aber haben mit der Benennung und Anwendung eines unabhängigen Gerechtigkeitsmaßstabes Probleme: Sie wollen sich nicht über den demokratischen Souverän stellen und - quasi als Philosophenkönige - dekretieren, was gerecht und was ungerecht sei. Zugleich erkennen sie mit der Ablehnung eines einheitlichen und unabhängigen Gerechtigkeitsstandards den Wert- und Interessenpluralismus moderner Gesellschaften an.

Rawls selbst bestimmt sein Verständnis von Gerechtigkeit als Fairness letztlich substanziell und nicht prozedural (Rawls 1995, S. 170; Lafont 2003, S. 164); eine gerechte politische Ordnung könne zwar niemals dem Anspruch vollständiger Verfahrensgerechtigkeit genügen, müsse aber nach Möglichkeit unvollständige Verfahrensgerechtigkeit gewährleisten, wobei Gerechtigkeitsmaßstäbe für Verfahrensergebnisse unabhängig vom Verfahren selbst existieren (Rawls [1971] 1999, S. 173). Jürgen Habermas dagegen möchte sein Modell deliberativer Demokratie trotz seines epistemischen Anspruchs als Fall reiner Verfahrensgerechtigkeit verstanden wissen (Habermas 1999).

Solche reine Verfahrensgerechtigkeit liegt für Rawls dann vor, wenn die Gerechtigkeit des Ergebnisses mit der Gerechtigkeit des Verfahrens zusammenfällt, wenn also kein verfahrensunabhängiger Maßstab für die Bewertung von Verfahrensergebnissen vorliegt (Rawls [1971] 1999, S. 86). Klassisches Beispiel für reine Verfahrensgerechtigkeit sei die Lotterie: Ob der Gewinner seinen Gewinn ,verdient' hat, ob er ein guter Mensch oder bereits Millionär ist, ist für die Bewertung des Ergebnisses irrelevant. Entscheidend ist allein, dass das Verfahren gerecht war, dass also jedes Los die gleiche Gewinnchance hatte. Dabei handelt es sich bei der Gleichheit der Gewinnchancen, auf der die Gerechtigkeit des Verfahrens beruht, durchaus um ein substanzielles Kriterium, das sich jedoch ausschließlich auf das Verfahren selbst, nicht auf seine Ergebnisse beziehen lässt. Anfechten lässt sich das Ergebnis einer Lotterie daher nur über den Nachweis von Verfahrensfehlern, nicht aufgrund einer an irgendwelchen anderen Maßstäben (wie Bedarf, Verdienst oder Pareto-Effizienz) gemessenen Ungerechtigkeit seiner Ergebnisse: Wie die voll- 
ständige Verfahrensgerechtigkeit garantiert auch die reine Verfahrensgerechtigkeit die Gerechtigkeit der Verfahrensergebnisse (vgl. Lafont 2003, S. 165). ${ }^{9}$

Auch in der klassischen vertragstheoretischen Begründung der Demokratie lässt sich das Verfahren freier Wahlen, in denen jeder Bürger Stimmrecht und jede Stimme dasselbe Gewicht hat, als Fall reiner Verfahrensgerechtigkeit begreifen. Während in der Lotterie jedoch jeder Teilnehmer mit dem Kauf eines Loses dem Verfahren faktisch zustimmt, kann die Zustimmung zum demokratischen Verfahren zur Setzung allgemeinverbindlicher Normen nur unterstellt oder gar als hypothetische gefasst werden. Wenn man aber für das Verfahren der demokratischen Mehrheitsentscheidung Zustimmung unterstellen oder hypothetisch voraussetzen kann, so ist dies prinzipiell auch für andere Verfahren der politischen Entscheidungsfindung denkbar.

\section{Verfahrensgerechtigkeit in der Verteilung von Gesundheitsgütern}

Welche Konzeption von Verfahrensgerechtigkeit bietet sich nun für Entscheidungen über die Verteilung von Gesundheitsgütern an? Die Idee vollständiger Verfahrensgerechtigkeit, welche die Vorstellung beinhaltet, gerechte Entscheidungen ließen sich aus übergeordneten, abstrakten Prinzipien ableiten, ist, wie oben argumentiert und von Holm (Holm 2000) am Beispiel der nordischen Länder eindringlich illustriert, an der Komplexität konkreter empirischer Entscheidungen gescheitert. In Theorie und Praxis einflussreich und plausibel zu begründen sind hingegen Modelle der deliberativen Entscheidungsfindung (Fleck 1994; Dworkin 2000; Daniels u. Sabin 2002). Wie ich im Folgenden anhand des berühmtesten Modells deliberativer Entscheidungsfindung in der Verteilung von Gesundheitsgütern - dem von Norman Daniels und James E. Sabin entwickelten Modell „Accountability for Reasonableness“ (AFR) - darlegen möchte, bleibt das Verhältnis von prozeduraler zu substanzieller Gerechtigkeit dabei jedoch unterbestimmt. Denn wenn hier von prozeduraler Gerechtigkeit die Rede ist, dann ist zumeist beides zugleich gemeint: die demokratische Legitimation im Sinne reiner Verfahrensgerechtigkeit und die Legitimation durch eine faire Prüfung von Evidenz und Argumenten im Sinne unvollständiger Verfahrensgerechtigkeit, ähnlich wie bei einem fairen Gerichtsverfahren.

Der Philosoph Norman Daniels und der Internist und Bioethiker James E. Sabin haben ihr Modell „Accountability for Reasonableness“, das als Referenzmaßstab für die Bewertung von Entscheidungsverfahren in der Verteilung knapper Gesundheitsgüter dienen soll, anhand einer empirischen Untersuchung solcher Verfahren bei amerikanischen Krankenversicherungen entwickelt (Daniels u. Sabin 2002). Die Entscheidungen, um die es in diesen Fällen ging, waren also zunächst keine politischen Entscheidungen, sondern Entscheidungen innerhalb privater (gewinnorientierter oder freigemeinnütziger) Unternehmen. Die Legitimation dieser Entscheidungen über demokratische Mehrheiten oder auf solchen

9 Ein Beispiel für die - politische - Verteilung eines knappen Gutes durch Lotterie sind die Lotterien, die in den Vereinigten Staaten bei der Vergabe von Greencards teilweise durchgeführt werden. Zur möglichen demokratischen Funktion von Lotterien, siehe Buchstein 2009. 
basierenden Institutionen stand also de facto gar nicht zur Debatte - auch wenn sich normativ die Frage stellt, ob die Bedingungen und der Leistungsumfang privater Krankenversicherungen nicht auch politisch reguliert werden sollten. Dieser Entstehungszusammenhang des Modells von Daniels und Sabin erklärt, weshalb "Accountability for Reasonableness“ den Aspekt der mehrheitsdemokratischen Legitimation auszublenden scheint und die dort benannten Kriterien eher denen eines fairen Gerichtsverfahrens gleichen. In seinem jüngsten Buch lehnt Daniels mehrheitsdemokratische Entscheidungen über die Verteilung von Gesundheitsleistungen jedoch auch explizit ab (Daniels 2008, S. 111-113).

Das Modell „Accountability for Reasonableness“ soll Entscheidungsträger dazu zwingen, Wertentscheidungen explizit zu machen und die institutionalisierte Entscheidungsfindung mit einem breiten deliberativ-demokratischen Willensbildungsprozess verbinden (Daniels 2008, S. 118-119). Es benennt vier Bedingungen eines gerechten Verfahrens (vgl. ebd.):

Öffentlichkeit: Entscheidungen und ihre Begründungen (nicht aber der Prozess der Entscheidungsfindung selbst!) müssen öffentlich zugänglich sein.

Relevante Gründe: Entscheidungen müssen auf Gründen basieren, die von gerecht denkenden Menschen („fair minded people“) als relevant anerkannt werden. Alle relevanten Gründe sollten im Entscheidungsprozess berücksichtigt werden, was Daniels und Sabin durch die Beteiligung verschiedener Gruppen von Betroffenen („stakeholder“) sicherstellen wollen.

Revision und Einspruch: Es muss die Möglichkeit geben, gegen ergangene Entscheidungen Einspruch einzulegen und eine Revision „in the light of new evidence and arguments" (Daniels 2008, S. 119) zu bewirken.

Verbindliche Regulierung: Durch verbindliche Regulierung ist sichergestellt, dass das angewandte Verfahren den Bedingungen 1-3 entspricht.

Daniels sieht AFR als Fall reiner Verfahrensgerechtigkeit an (2008, S. 109). Wie er selbst feststellt, stimmen die in AFR benannten Bedingungen eines gerechten Verfahrens weitgehend, wenn auch nicht vollständig, mit den von Gutmann und Thompson benannten Prinzipien deliberativer Demokratie - Öffentlichkeit, Reziprozität und Rechenschaftspflicht (accountability) - überein (Daniels 2008, S. 119, Fn. 10; Gutmann u. Thompson 1996). Zentral ist offensichtlich die Bedingung der „relevanten Gründe“, die Daniels zufolge der Reziprozitätsbedingung bei Gutmann und Thompson entspricht und auf eine Beziehung zielt, in der ein „Recht auf Rechtfertigung“ zum Tragen kommt (Forst 2007). Die Bedingungen der Öffentlichkeit, Revision und verbindlichen Regulierung sind letztlich instrumentell zur Verwirklichung der Relevanzbedingung.

Was aber genau sind die relevanten Gründe, die rechtschaffene Menschen („fair-minded people“) akzeptieren können? Wie Rid darlegt, führt Daniels mit der Relevanzbedingung gewissermaßen durch die Hintertür ein substanzielles Kriterium ein: Gründe können nur in Hinblick auf ein implizit unterstelltes Kriterium mehr oder weniger relevant sein (Rid 2009, S. 13). Bei Daniels ist dieses Kriterium der zentrale Bezugspunkt seiner Theorie gerechter Gesundheitsversorgung: das Rawlsianische Prinzip der fairen Chancengleichheit (fair equality of opportunity). Die Tatsache, dass das Prinzip der fairen Chancengleichheit zu unbestimmt ist, um Priorisierungs- und Rationierungsprobleme in der Gesundheitsversorgung 
zu lösen, bringt Daniels (mit Sabin) überhaupt erst dazu, AFR als Vorschlag eines gerechten Verfahrens in Konflikten um die Verteilung knapper Güter zu entwickeln (vgl. Rid 2009, S. 14). Zugleich behält das Prinzip der fairen Chancengleichheit als Maßstab seine Geltung, was auch Daniels' Formulierung der Revisionsbedingung deutlich macht (s. o.): Entscheidungen sollen nicht nur aufgrund von Verfahrensfehlern revidierbar sein - wie dies in Fällen reiner Verfahrensgerechtigkeit, etwa im Beispiel der Lotterie, der Fall ist. Vielmehr können auch neue Argumente, mit anderen Worten: neue Gründe, die sich in Hinblick auf das Kriterium der fairen Chancengleichheit als relevant herausgestellt haben, eine Revision von Entscheidungen rechtfertigen.

Rid schlägt vor, den Widerspruch in Daniels' Argumentation in einem Konzept eingeschränkter reiner Verfahrensgerechtigkeit („,constrained pure procedural justice“) aufzulösen. Die Idee dabei ist, dass sich aus dem Prinzip der fairen Chancengleichheit gerechte Lösungen zwar nicht unmittelbar ableiten lassen, dieses wohl aber einen Raum gerechter Lösungen bestimmt, innerhalb dessen die Gerechtigkeit der Entscheidung für eine konkrete Lösung auf reiner Verfahrensgerechtigkeit beruht (Rid 2009, S. 15-16). Dieser Vorschlag steht in bemerkenswertem Einklang mit den Vorstellungen vieler deliberativer Demokratietheoretiker, die sich einerseits auf reine Verfahrensgerechtigkeit berufen und andererseits davon ausgehen, dass ein faires, deliberatives Verfahren keine Ergebnisse produzieren wird, die seine eigenen Funktionsbedingungen gefährden könnten - was eine extrem ungleiche und als ungerecht empfundene Verteilung wichtiger Güter wohl tun würde.

Können wir also für Verteilungsentscheidungen in der Gesundheitsversorgung einerseits das Prinzip fairer Chancengleichheit als Maßstab für faire Entscheidungen anerkennen und andererseits auf ein Verfahren setzen, das „eingeschränkte reine Verfahrensgerechtigkeit" generiert? Nein, denn das Prinzip fairer Chancengleichheit bleibt, auch wenn es sich überaus plausibel rechtfertigen lässt, in modernen, pluralistischen Gesellschaften nur eines unter vielen Gerechtigkeitsprinzipien, dessen Geltung kontingent ist und sich letztlich nur durch demokratische Entscheidungen legitimieren lässt - die auf reiner Verfahrensgerechtigkeit beruhen müssen. Daniels' Präferenz für das Prinzip der fairen Chancengleichheit bleibt, auch wenn sie von vielen Menschen geteilt wird, partikular. Er kann als Philosoph und Gerechtigkeitstheoretiker für die Anwendung eines bestimmten Verteilungsprinzips plädieren, aber politisch und als Demokrat nicht verlangen, dass dieses Prinzip einer Gesellschaft aufgezwungen wird. Wenn wir in einer Demokratie ein Verfahren durchsetzen, das eigenen, partikularen Gerechtigkeitsmaßstäben dient, dann kann dieses Verfahren eben nicht mehr auf reine Verfahrensgerechtigkeit bauen, weil es dann tendenziell den Interessen derjenigen Personen und Gruppen dient, die diesen einen Maßstab gegenüber anderen möglichen befürworten. ${ }^{10}$

10 Eine Möglichkeit der Eingrenzung des Raumes gerechter Lösungen bestünde jedoch darin, Lösungen deshalb auszuschließen, weil sie die Voraussetzungen eines gerechten Verfahrens selbst unterminieren. Aufgrund der zentralen Rolle, welche die Absicherung gesundheitlicher Lebensrisiken für die effektive Nutzung demokratischer und freiheitlicher Rechte besitzt, erscheint dies durchaus plausibel. Die Frage, inwieweit eine solche Einschränkung gesellschaftlich konsensfähig ist (denn Konsensund nicht nur Mehrheitsfähigkeit wäre hier gefragt), bleibt jedoch letztlich eine empirische. 
Daniels scheint diese Problematik einzugestehen, wenn er darauf beharrt, dass er selbst AFR als Fall reiner Verfahrensgerechtigkeit versteht, es zugleich aber denjenigen, die auf der transzendentalen, also verfahrensunabhängigen Geltung bestimmter Prinzipien beharren, auch als Fall unvollständiger Verfahrensgerechtigkeit anbietet: „The fair process I seek will turn out to be pure as long as we have no consensus on fine-grained distributive principles; it will be imperfect at best for anyone who insists that some particular principle tells us what the just outcome should be" (Daniels 2008, S. 109, Fn. 5). Politisch ist Daniels also auf die reine Verfahrensgerechtigkeit zurückgeworfen. Als Gerechtigkeitstheoretiker kann er darauf hoffen, dass das Verfahren Ergebnisse produziert, die seinen Gerechtigkeitsvorstellungen und bevorzugten Verteilungsprinzipien entsprechen aber diese Hoffnung müssen in der Demokratie eben auch jene haben können, die andere Gerechtigkeitsvorstellungen haben und das Prinzip fairer Chancengleichheit nicht als Maßstab gerechter Verteilungsergebnisse anerkennen.

\section{Gerechtes Institutionendesign}

Es geht in der Verteilung knapper Gesundheitsgüter also - zumindest aus politikund demokratietheoretischer Sicht - um die Verwirklichung von Verfahrensgerechtigkeit im Sinne von Rawls' reiner prozeduraler Gerechtigkeit. Wie aber könnte ein Verfahren aussehen, das von den Adressaten seiner Ergebnisse als gerecht angesehen wird? Die Kriterien der „Accountability for Reasonableness“ bieten hier als substanzielle und normativ gehaltvolle Kriterien der Verfahrensauswahl (nicht der Ergebnisauswahl!) durchaus wichtige Anhaltspunkte. Möchte man das Verfahren zudem als demokratisches qualifizieren, so kommen, auf der Meta-Ebene der Verfahrensauswahl und zur Ausgestaltung des Verfahrens selbst weitere Kriterien hinzu: Neben der Rechenschaftspflicht (accountability) wäre etwa eine demokratische Autorisierung der Entscheidungsträger und unter Umständen auch eine Möglichkeit der Abwahl (im Sinne eines Recall) gefordert.

Zugleich muss ein Verfahren, das von den Bürgern einer Demokratie als gerecht anerkannt wird, nicht zwangsläufig ein mehrheitsdemokratisches sein. Die Einwände, die Daniels gegen rein aggregativ-mehrheitsdemokratische Entscheidungen in der Verteilung von Gesundheitsgütern vorbringt (Daniels 2008, S. 111113), dürften durchaus von einer Mehrheit der Bevölkerung geteilt werden: Mehrheitsentscheidungen können Wähler und Repräsentanten dazu veranlassen, sich an kurzfristigen materiellen oder parteipolitischen Interessen zu orientieren, und eine starke Politisierung und Emotionalisierung von Rationierungsentscheidungen könnte zu mangelhaft informierten und begründeten, kurzsichtigen Entscheidungen oder zu einer Tyrannei der Mehrheit führen. Umfragen zeigen, dass die Mehrzahl der Bürger in entwickelten Demokratien sich mit der Komplexität von Priorisierungs- und Rationierungsentscheidungen in der Gesundheitsversorgung überfordert sieht und eine zumindest teilweise Delegation von Entscheidungskompetenzen an Experten befürwortet (Lomas 1997). Dennoch geht der befürwortete Verzicht auf unmittelbare mehrheitsdemokratische Kontrolle von Entscheidungsverfahren mit Erwartungen an Transparenz, deliberative Qualität und Einspruchsmöglichkeiten einher, die im Wesentlichen den in AFR formulier- 
ten Kriterien entsprechen und deren verbindliche Regulierung (im Sinne des vierten Kriteriums) sicherzustellen ist.

Nun ist aber eine Vielzahl institutioneller Lösungen denkbar, die solchen Erwartungen beziehungsweise den in AFR formulierten Bedingungen genügen können - wie sollen wir uns zwischen ihnen entscheiden? Dies ist die zentrale Frage, die bei Daniels und Sabin unbeantwortet bleibt. So wie das abstrakte Prinzip fairer Chancengleichheit zu unbestimmt ist, um zwischen mehreren konkreten Verteilungslösungen zu entscheiden, so sind die Prinzipien eines fairen, deliberativen Verfahrens, die Daniels und Sabin benennen, zu unbestimmt, um aus ihnen die Entscheidung für ein konkretes Verfahren abzuleiten. Gleiches gilt für Kriterien demokratischer Qualität - auch diese sind für die Auswahl und Gestaltung konkreter Verfahren nicht hinreichend. So wie sich aus dem Prinzip der Mehrheitsherrschaft keine Entscheidung für ein bestimmtes Wahlrecht ableiten lässt, lässt sich aus dem Prinzip der Reziprozität - oder anderen Prinzipien deliberativer Entscheidungsfindung, wie dem Prinzip der relevanten Gründe oder dem Recht auf Rechtfertigung - kein konkretes Institutionendesign ableiten. Während für Daniels offenbar jedes Verfahren, das den Bedingungen von AFR genügt, gleichermaßen fair ist und zu gleichermaßen akzeptablen Ergebnissen führt, muss eine politiktheoretisch informierte Sichtweise davon ausgehen, dass Verfahrensentscheidungen - und zwar auch solche für demokratische und deliberative Verfahren - Folgen für die resultierende Verteilung haben und damit selbst schon Verteilungsentscheidungen beinhalten (siehe Scharpf 1989).

Auch wenn sich Entscheidungsverfahren zur Verteilung knapper Ressourcen letztlich nur über das Konzept der reinen prozeduralen Gerechtigkeit begründen lassen, müssen die Ergebnisse dieser Verfahren letztlich die empirisch gegebenen, wenn auch pluralen, oft widersprüchlichen und praktisch schwer zu ermittelnden Gerechtigkeitsvorstellungen ihrer Adressaten widerspiegeln. Wenn sie dem Gerechtigkeitsempfinden einzelner Mitglieder der Gemeinschaft eklatant widersprechen oder nicht in Einklang mit dem Empfinden der Mehrheit stehen, müssen durch als gerecht anerkannte Verfahren getroffene Rationierungsentscheidungen - wie alle demokratischen Entscheidungen - zwar zunächst einmal akzeptiert werden, aber prinzipiell revidierbar sein. Während diese Revidierbarkeit von Entscheidungen auch bei Daniels und Sabin explizit eingefordert wird, folgt aus der Verfahrensabhängigkeit von Verteilungsergebnissen, dass auch das Verfahren selbst politisierbar und revidierbar sein muss: nämlich dann, wenn es entweder systematisch Ergebnisse produziert, die den Gerechtigkeitsvorstellungen seiner Adressaten widersprechen, oder dann, wenn es bestimmte Gruppen dauerhaft und in ungerechtfertigter Weise zu Verlierern macht.

Die Delegation von Entscheidungen an spezialisierte Gremien kann durchaus in dem Sinne legitim sein, dass sie von einer Bevölkerungsmehrheit nicht nur akzeptiert, sondern sogar eingefordert wird. Wie aber sieht es mit Entscheidungen auf der Metaebene aus, also Entscheidungen darüber, wie man entscheidet? Können auch diese im Zweifelsfall an Experten oder andere, nicht-demokratisch autorisierte Akteure delegiert werden? Aus demokratietheoretischer Sicht ist die Entscheidung darüber, wie man entscheidet, gewissermaßen ein Akt der Verfassungsgebung, mit dem über grundlegende Prinzipien des Zusammenlebens ent- 
schieden wird, welche dann in untergeordneten Institutionen und Regeln fortgeschrieben werden. Auch wenn es im hier diskutierten Fall nur um die Ausgestaltung eines einzelnen Entscheidungsverfahrens innerhalb demokratischer Verfassungsstaaten geht, wird deutlich, dass mit der Entscheidungsebene auch die Anforderungen an direkte demokratische Autorisierung zunehmen. ${ }^{11}$ Auf der Metaebene der Verfahrensauswahl und -ausgestaltung haben Entscheidungen weit reichende Konsequenzen, denn hier werden letztlich die Parameter bestimmt, die Entscheidungen über die Finanzierung konkreter Gesundheitsleistungen zumindest in Teilen determinieren. Dieser Argumentation zufolge ist die Art reiner Verfahrensgerechtigkeit, die Verfahrensentscheidungen erfordern, nur durch mehrheitsdemokratische Verfahren zu realisieren. Das Verfahren, an das Entscheidungen delegiert werden, muss also seinerseits mehrheitsdemokratisch legitimiert sein, und es muss deutlich sein, dass die Verfahrensentscheidung durch eine mehrheitsdemokratische Entscheidung revidiert werden kann.

Wie die resultierenden Entscheidungen muss auch die Verfahrensauswahl im Einklang mit den Gerechtigkeitsvorstellungen ihrer Adressaten stehen. Gerechtigkeitsvorstellungen, die Institutionen über die Auswahl einzelner Merkmale eingeschrieben sind, müssen explizit gemacht werden. Konsensregelungen etwa können die Inklusivität der Entscheidungsfindung erhöhen, schützen zugleich aber häufig den Status quo und zementieren bestehende Ungleichheiten in der Ressourcenverteilung. Ein von Gesundheitsökonomen dominiertes Gremium wird Effizienzkriterien stärker gewichten als Bedarfskriterien und im Zweifel Entscheidungen treffen, die dem Gerechtigkeitsempfinden der Bevölkerung widersprechen - das Beispiel des US-Bundesstaats Oregon hat dies eindrücklich gezeigt (Hadorn 1991).

Es bedarf also für den demokratischen Prozess der Verfahrensauswahl weiterer Informationen über die Verteilungswirkung von Verfahren: Wenn eine Gesellschaft mehrheitlich bestimmte Verteilungsziele erreichen will, muss sie wissen, welche Institutionen hierfür instrumentell geeignet sind. Informationen über die Verteilungseffekte von Verfahren sind aber nicht nur wichtig, weil Verfahren und Ergebnisse in Einklang mit den Gerechtigkeitsvorstellungen ihrer Adressaten stehen müssen. Sie sind auch wichtig, um reine Verfahrensgerechtigkeit zu sichern: Nur wenn wir die Verteilungsfolgen von Verfahren kennen, sind wir in der Lage festzustellen, wer die Gewinner und Verlierer einer institutionellen Regelung sind und ob ein Verfahren durch einen bias in die eine oder andere Richtung als solches nicht (mehr) gerecht ist. ${ }^{12}$ Wenn die reine Verfahrensgerechtigkeit einer institutionellen Lösung in Frage steht, besteht in besonderer Weise Anlass, Verfahren und Gremien zu prüfen und neu zu gestalten.

Nun ließe sich einwenden, dass mit den Verteilungsfolgen von Entscheidungsverfahren erneut ein substanzielles Kriterium zur Anwendung kommt, welches im

11 Die Tatsache, dass viele Verfassungen, gerade in Situationen des Umbruchs und der Transformation zur Demokratie, gerade nicht demokratisch gesetzt wurden, ändert nichts an diesem normativen Anspruch.

12 Natürlich produziert jedes Verfahren, so etwa auch die Lotterie, Gewinner und Verlierer. Problematisch wird es dann, wenn es um systematische Gewinner und Verlierer geht, Verlierer also nicht Verlierer des Verfahrens selbst, sondern der Verfahrensentscheidung sind: wenn also z. B. an Menschen über 60 prinzipiell nur Nieten verkauft werden. 
Widerspruch zum Prinzip der reinen Verfahrensgerechtigkeit steht. Die Verteilungseffekte eines Verfahrens sind jedoch nicht als übergeordnetes Bewertungskriterium, sondern allein als Information von Bedeutung. Auch der Käufer eines Loses möchte wissen, wie die Gewinne der Lotterie verteilt sind: Gibt es beispielsweise einen einzigen Hauptgewinn oder viele Gewinne in unterschiedlichen Gewinnklassen? Angesichts der Tatsache, dass ein vollkommen neutrales Entscheidungsverfahren, anders als eine vollkommen faire Lotterie, kaum denkbar und praktisch nicht zu konstruieren ist, gewinnen solche Informationen zusätzlich an Relevanz - und zwar für diejenigen, die die Verfahrensentscheidung treffen, nämlich die Bürger eines Gemeinwesens.

Wenn man die Rolle der inklusiven, mehrheitsdemokratischen Entscheidungsfindung von der substanziellen Ebene (wer soll welche Leistungen erhalten?) auf die Meta-Ebene des Verfahrens verlagert (wie soll darüber entschieden werden, wer welche Leistungen erhält?), muss man aus prinzipiellen Gründen auch die darüber liegende Entscheidungsebene (wie und wer soll darüber entscheiden, wie darüber entschieden wird, wer welche Leistungen erhält?) thematisieren. Denn auch die Verfahrensentscheidung wird nicht direkt demokratisch, sondern von demokratisch gewählten Repräsentanten getroffen, wobei bereits grundsätzliche Entscheidungen über Regierungssystem und Wahlrecht tendenziös sein und reine demokratische Verfahrensgerechtigkeit unterminieren können. Hier gelangt man tatsächlich auf die Ebene der Verfassungsgebung und zu der Frage, wie wir ohne Rückgriff auf bestehende Verfahren diese selbst reformieren können - ein Regressproblem.

Auch wenn dieses Regressproblem letztlich nicht auflösbar ist, ist die Problematisierung von Verfahrensentscheidungen durch politische Entscheidungsträger und in einem öffentlichen Diskurs von großer Bedeutung, um auf der substanziellen Ebene sicherzustellen, dass Verteilungsergebnisse in Einklang mit Gerechtigkeitsvorstellungen ihrer Adressaten stehen und auf der prozeduralen Ebene reine demokratische Verfahrensgerechtigkeit zu sichern. Neben der Anforderung demokratischer Legitimation lässt sich auch die von Daniels und anderen erhobene Forderung nach einem deliberativen Austausch von Gründen und einer eingehenden Prüfung vorhandener Informationen als substanzielles Kriterium für die Verfahrensauswahl gut begründen. Doch auch wenn für die Auswahl von Verfahren substanzielle Kriterien zu bestimmen sind, bleibt die Form der Gerechtigkeit, um die es hier geht, reine Verfahrensgerechtigkeit - denn die benannten Kriterien richten sich ausschließlich auf das Verfahren selbst, nicht aber auf seine Ergebnisse. In jedem Fall sollten Verfahrensentscheidungen daher politisiert, durch empirische Erkenntnisse über Verfahrenswirkungen informiert und deliberativ getroffen werden.

\section{Fazit}

Gesundheit ist ein Gut mit besonderen, weil konditionalen Qualitäten, aus denen sich starke Argumente für die Sicherstellung einer, zumindest grundlegenden, Gesundheitsversorgung als öffentliches Gut ableiten lassen. Wie ich im zweiten Abschnitt dieses Papiers argumentiert habe, haben aber auch die Güter, die zur 
Erhaltung und Wiederherstellung von Gesundheit erforderlich sind, besondere Merkmale: Sie sind zum einen nur begrenzt teilbar und zum anderen heterogene Güter. Diese Eigenschaften führen zu Problemen bei ihrer Allokation unter Knappheitsbedingungen. Gesundheitsgüter waren immer tendenziell knappe Güter, aber der technologische Fortschritt hat im Gesundheitssektor in den letzten Jahrzehnten zu einer Dynamik der Ausgabensteigerung geführt, die Grenzziehungen, und damit die Rationierung von Gesundheitsleistungen, notwendig zu machen scheint.

Im dritten Abschnitt habe ich Versuche diskutiert, Probleme bei der Allokation knapper Gesundheitsgüter durch den Rückgriff auf abstrakte Gerechtigkeitsprinzipien zu lösen. Ich habe gezeigt, warum dieser Versuch der Herausforderung nicht gerecht werden konnte, die die Gewichtung konfligierender Prinzipien und die Kontextualisierung, also Anwendung von Prinzipien in konkreten Fällen, darstellen. Empirisch ist dieser Versuch, wie das Beispiel der Prinzipienkommissionen zeigt, gescheitert - und er musste scheitern, weil sich politische Entscheidungen nicht durch die Anwendung von Prinzipien ersetzen lassen.

Individuelle substanzielle Gerechtigkeitsvorstellungen spielen bei der Verteilung von Gesundheitsgütern zwar insofern eine Rolle, als dass sie für die Bürger Maßstab zur Bewertung von Verteilungsentscheidungen sind. Wie ich in Abschnitt 4 argumentiert habe, lässt sich Gerechtigkeit in entsprechenden Verteilungskonflikten aus politiktheoretischer Sicht aber nur als prozedurale Gerechtigkeit begreifen. Anhand von Rawls' Unterscheidung zwischen vollständiger, unvollständiger und reiner Verfahrensgerechtigkeit habe ich dargelegt, dass es hier um reine Verfahrensgerechtigkeit gehen muss. Dies bedeutet, dass die Gerechtigkeit der Entscheidung ausschließlich aus der Gerechtigkeit des Verfahrens resultiert und auf einen unabhängigen moralischen Maßstab zur Bewertung von Entscheidungen zwar keinesfalls auf der individuellen, wohl aber auf der politischen Ebene in modernen Demokratien verzichtet werden muss.

Im letzten Abschnitt habe ich dann diskutiert, was hieraus für ein gerechtes Institutionendesign folgt. Zentral ist hier zunächst die Feststellung, dass zur Bewertung institutioneller Lösungen Informationen erforderlich sind: Informationen über die Verteilungsfolgen von Entscheidungsverfahren. Diese Informationen sind bedeutsam, um einerseits sicherzustellen, dass die Ergebnisse eines Verfahrens weitgehend in Einklang mit den Gerechtigkeitsvorstellungen der Bevölkerung stehen, und um andererseits reine Verfahrensgerechtigkeit dadurch zu sichern, dass dauerhafte Verlierer identifiziert und Verfahren entsprechend revidiert werden können.

Wenn wir aber davon ausgehen müssen, dass Institutionen durch die Auswahl einzelner Verfahrensmerkmale immer auch substanzielle Gerechtigkeitsvorstellungen eingeschrieben werden, wie ist dann reine Verfahrensgerechtigkeit in Verteilungskonflikten sicherzustellen? Institutionen können und müssen nicht normfrei sein. Wichtig ist, dass das Design mit Entscheidungen beauftragter Gremien politisiert und mehrheitsdemokratisch legitimiert wird. Verfahrensentscheidungen sind, zumindest in Teilen, immer auch selbst schon Verteilungsentscheidungen. Wer dauerhaft zu den Verlierern einer solchen Entscheidung zählt, muss das Verfahren öffentlich anfechten können und wissen wann, wie und durch wen es 
revidiert werden kann. Dies gilt nicht nur für Entscheidungsverfahren in der Allokation von Gesundheitsleistungen, sondern für alle demokratischen Entscheidungsverfahren - nicht nur, aber gerade dann, wenn es um die Verteilung essenzieller und knapper Güter geht.

\section{Literatur}

Aaron, Henry, und William B. Schwartz. 1990. Rationing Health Care: The Choice Before Us. Science 247:418-422.

Becker, Irene, und Robert Hauser. 2004. Soziale Gerechtigkeit - eine Standortbestimmung. Berlin: Edition Sigma.

Buchstein, Hubertus. 2009. Bausteine einer aleatorischen Demokratietheorie. Losverfahren in der modernen Demokratie. In ders.: Demokratietheorie in der Kontroverse, 253280. Baden-Baden: Nomos.

Calabresi, Guido, und Philip Bobbit. 1978. Tragic Choices. New York: Norton.

Daniels, Norman. 2008. Just Health. Meeting Health Needs Fairly. Cambridge: Cambridge University Press.

Daniels, Norman, und James E. Sabin. 2002. Setting Limits Fairly. Can We Learn to Share Medical Resources? Oxford: Oxford University Press.

Dworkin, Ronald. 2000. Justice and the High Cost of Health. In ders.: Sovereign Virtue, 307-319. Cambridge: Harvard University Press.

Elster, Jon. 1995. Introduction: The Idea of Local Justice. In Local Justice in America, Hrsg. Jon Elster, 1-24. New York: Russell Sage Foundation.

Estlund, David. 1993. Making Truth Safe for Democracy. In The Idea of Democracy, Hrsg. David Copp, Jean Hampton, und John E. Roemer, 71-100. Cambridge: Cambridge University Press.

Fleck, Leonard M. 1994. Just Caring - Oregon, Health-Care Rationing, And Informed Democratic Deliberation. Journal of Medicine and Philosophy 19:367-388.

Forst, Rainer. 2007. Das Recht auf Rechtfertigung. Frankfurt a. M.: Suhrkamp.

Goodin, Robert E., und David Estlund. 2004. The persuasiveness of democratic majorities. Politics, Philosophy \& Economics 3:131-142.

Gosepath, Stefan. 2007. Kann das Gut Gesundheit gerecht verteilt werden? In Gesundheit für alle - wie lange noch? Rationierung und Gerechtigkeit im Gesundheitswesen, Hrsg. Nationaler Ethikrat, 19-34. Berlin: Nationaler Ethikrat.

Greven, Michael Th. 1999. Die politische Gesellschaft. Kontingenz und Dezision als Probleme des Regierens in der Demokratie. Opladen: Leske + Budrich.

Gutmann, Amy, und Dennis Thompson. 1996. Democracy and Disagreement. Cambridge: Belknap Press.

Habermas, Jürgen. 1999. Richtigkeit vs. Wahrheit. Zum Sinn der Sollgeltung moralischer Aussagen und Normen. In ders.: Wabrheit und Rechtfertigung, 271-318. Frankfurt a. M.: Suhrkamp.

Hadorn, David C. 1991. Setting Health Care Priorities in Oregon: Cost-Effectiveness Meets the Rule of Rescue. JAMA 265:2218-2225.

Holm, Søren. 2000. Developments in the Nordic countries - goodbye to the simple solutions. In The Global Challenge of Health Care Rationing, Hrsg. Angela Coulter und Chris Ham, 29-37. Buckingham/Philadelphia: Open University Press. 
Kliemt, Hartmut. 2003. Rationierung im pluralen Rechtsstaat. In Gesundheitsversorgung im Alter, Hrsg. Georg Marckmann, 59-71. Stuttgart: Schattauer.

Kühn, Hagen. 2004. Demographischer Wandel und demographischer Schwindel. Zur Debatte um die gesetzliche Krankenversicherung. Blätter für deutsche und internationale Politik 49:742-751.

Lafont, Christina. 2003. Procedural justice? Implications of the Rawls-Habermas Debate for Discourse Ethics. Philosophy and Social Criticism 29:163-181.

Liebig, Stefan. 2008. Was versteht man unter sozialer Gerechtigkeit? Perspektiven und Befunde der empirischen Gerechtigkeitsforschung. Gesellschaft - Wirtschaft - Politik 57:495-506.

Liebig, Stefan, und Maike May. 2009. Dimensionen sozialer Gerechtigkeit. Aus Politik und Zeitgeschichte 47:3-8.

List, Christian, und Robert E. Goodin. 2001. Epistemic Democracy: Generalizing the Condorcet Jury Theorem. The Journal of Political Philosophy 9:277-306.

Lomas, Jonathan. 1997. Reluctant Rationers: Public Input to Health Care Priorities. Journal of Health Service Research and Policy 2:103-111.

Marshall, Thomas H. [1949] 1992. Staatsbürgerrechte und soziale Klassen. In ders.: Bürgerrechte und soziale Klassen, 33-49. Frankfurt a. M.: Campus Verlag.

McKie, John, und Jeff Richardson. 2003. The Rule of Rescue. Social Science \& Medicine 56:2407-2419.

Miller, David. 1999. Principles of Social Justice. Cambridge: Harvard University Press.

Nussbaum, Martha. 2006. Frontiers of Justice. Disability, Nationality, Species Membership. Cambridge: Belknap Press.

Rawls, John. 1995. Reply to Habermas. Journal of Philosophy 92:132-180.

Rawls, John. [1971] 1999. A Theory of Justice. Cambridge: Belknap Press.

Rid, Annette. 2009. Justice and procedure: How does "accountability for reasonableness" result in fair limit-setting decisions? Journal of Medical Ethics 35:12-16.

Scharpf, Fritz W. 1989. Decision Rules, Decision Styles and Policy Choices. Journal of Theoretical Politics 1:149-176.

Walzer, Michael. 1984. Spheres of Justice. A Defense of Pluralism and Equality. New York: Basic Books.

\section{Autorenangaben}

Dr. Claudia Landwehr, Schumpeter Fellow,

Goethe-Universität Frankfurt am Main, Institut für Politikwissenschaft,

Robert-Mayer-Straße 5, 60054 Frankfurt am Main,

landwehr@em.uni-frankfurt.de 